\title{
Н.Г. Малич \\ МОДЕЛИРОВАНИЕ РАСПРЕДЕЛЕНИЯ НАПРЯЖЕНИЙ, ДЕЙСТВУЮЩИХ ПРИ РАЗРУШЕНИИ ПОРОДЫ В ДРОБИЛКАХ С УЧЕТОМ КОНТАКТНОГО ТРЕНИЯ
}

Аннотация. Приведены исследования распределения напряжений, действующих при разрушении крепких материалов в зоне контакта с бронеплитами в щековых дробилках со сложным движением щеки и с конусами в конусных дробилках. Проанализировано влияние направления сил контактного трения при сжатии на эффективность разрушения горной массы. Разработана математическая модель для определения напряженного состояния породы. Показана возможность управления режимами нагружения для повышения эффективности процесса дробления.

Ключевые слова: горная порода, разрушение, напряжения, моделирование, дробилки, контактное трени.

Постановка проблемы и задачи. Для разрушения породы в качестве породоразрушающих инструментов в щековых дробилках используются бронеплиты, в конусных дробилках разрушение происходит между движущимися конусами, которые при взаимодействии с породой создают в зоне контакта нормальные и тангенциальные напряжения [1].

Характер распределения напряжений в породе при контакте с разрушающим инструментом имеет определяющее значение для процесса эффективного разрушения [2].

Поэтому исследование напряжённого состояния зон силового контакта инструмента с породой является актуальным.

В статье поставлена задача разработать математическую модель механизма разрушения для определения области зарождения трещин по которым проходит сжатие и сдвиг породы при ее разрушении в щековых дробилках со сложным движением щеки и в конусных дробилках, которые можно рассматривать как результат развития щековой дробилки,

(C) Малич Н.Г., 2019 
«Системні технологіï» 5 (124) 2019 «System technologies»

связанный с переходом от плоского к пространственному движению дробящего органа [3].

Методика исследований. В щековой дробилке со сложным движением щеки перемещение подвижной щеки происходит по эллиптическим траекториям, поэтому изменение величины контактного давления, возникающего при соприкосновении бронеплит с породой, изменяется также по эллиптическому закону (рис. 1а). В то же время силы контактного трения между бронеплитами направлены в разные стороны (рис.1б).

Анализируя распределение нагрузки по дробящим плитам по данным экспериментальных исследований, проведённых различными авторами [4,5], можно допустить, что при работе щековых дробилок распределение нагрузок вдоль рифления плиты близко к равномерному, а равнодействующая нагрузок условно может быть приложена к середине плиты и направлена перпендикулярно к ней.

Рассмотрим взаимодействие дробящих элементов (рифления бронеплит) в случае приложения внешних сил Q, направленных по нормали к линии контакта поверхности рифления с разрушаемыми материалами при равномерном распределении вдоль линии контакта касательных нагрузок Q1 [1,2] (Рис.2).

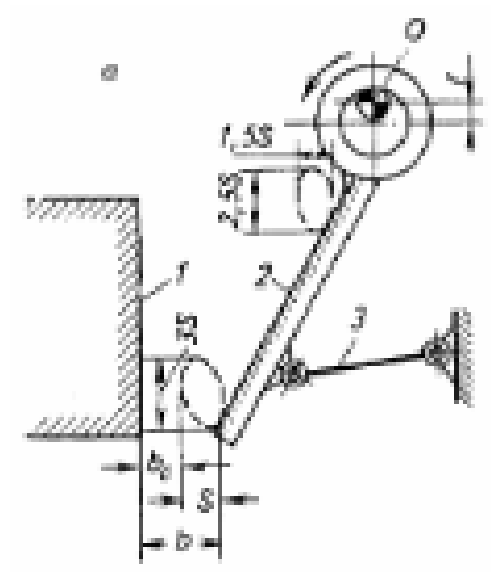

a

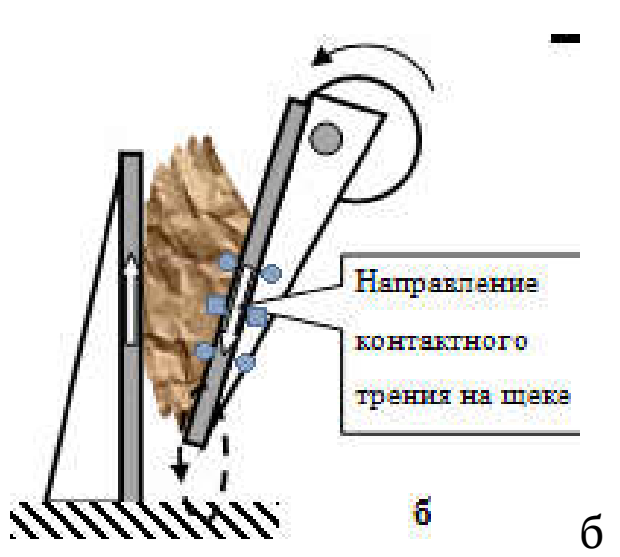

Рисунок 1 - Схема дробилки со сложным движением щеки:

а) 1-неподвижная щека, 2-подвижная щека, s - горизонтальная составляющая хода; б) схема направления сил контактного трения 


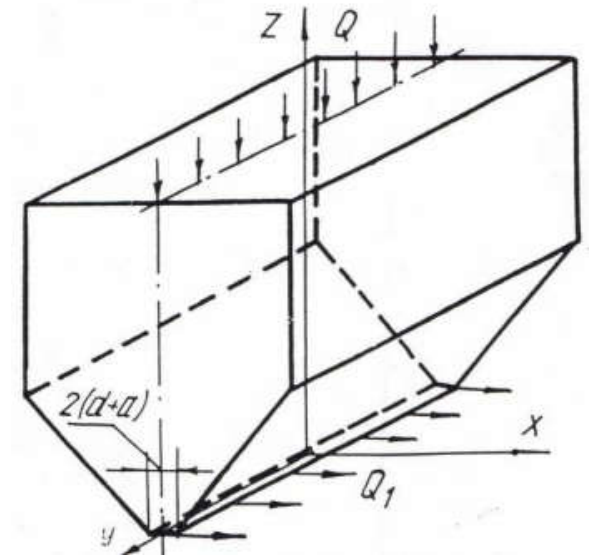

Рисунок 2 - Расчётная схема приложения загрузки

Решение поставленной задачи связано с существенными математическими трудностями [6-8]. Поэтому воспользуемся приближенным решением подобной задачи, полученным $[4,8]$ с учётом следующих допущений: контактное давление р распределяется по эллипсу в зоне соприкосновения поверхностей; касательные контактные нагрузки q пропорциональны нормальным.

$$
\frac{Q_{1}}{Q}=\frac{q_{\max }}{p_{\max }}=\varphi=\text { const }
$$

Напряжённое состояние породы определяется значением компонентов тензора напряжений:

$$
\begin{gathered}
\sigma_{x}=q_{\max }\left(2 e^{-a} \cos \beta_{0}-\operatorname{Sh} \alpha \sin \beta_{0} \frac{\sin 2 \beta_{0}}{\operatorname{Ch} 2 \alpha-\cos 2 \beta_{0}}\right) ; \\
\sigma_{y}=2 \mu q_{\max } e^{-\alpha} \cos \beta_{0} ; \\
\sigma_{z}=q_{\max } \operatorname{Sh} \alpha \sin \beta_{0} \sin 2 \frac{\beta_{0}}{\operatorname{ch} 2 \alpha-\cos 2 \beta_{0}} ; \\
\tau_{x z}=q_{\max }\left(e^{-a} \cos \beta_{0}-\operatorname{Sh} \alpha \sin \beta_{0}\left(1-\frac{\operatorname{Sh} 2 \alpha_{0}}{\operatorname{Ch} 2 \alpha-\cos 2 \beta_{0}}\right)\right) .
\end{gathered}
$$

здесь бу найдено из условия плоской деформации: $\sigma_{\mathrm{y}}=\mu\left(\sigma_{\mathrm{x}}+\sigma_{\mathrm{z}}\right) ; \mu$ - коэффициент Пуассона; $\alpha$ и $\beta$ - эллиптические координаты, связь которых с прямоугольными координатами х и у посредством гиперболических и круговых функций осуществляется следующим образом:

$$
x=(a+d) \operatorname{Ch} \alpha \cos \beta_{0}, z=(a+d) \operatorname{Sh} \alpha \sin \beta_{0} .
$$

Проанализируем состояние породы в зоне контакта. При х=0 плоскость, перпендикулярная площадке контакта, проходит через ее се- 
редину, из предыдущего соотношения $\beta_{0}=0,5 \pi$ т.к. $\cos \beta_{0}=0$. Подставим полученное значение $\beta_{0}=0,5 \pi$ в уравнение (1), найдём:

$$
\begin{gathered}
\sigma_{x}=\sigma_{y}=\sigma_{z}=0 ; \tau_{x z}=q_{\max }\left[e^{-\alpha}-\operatorname{Sh} \alpha\left(1-\frac{\operatorname{Sh} 2 \alpha}{\operatorname{ch} 2 \alpha+1}\right)\right] \text { или } \\
\tau_{x z}=q_{\max }\left[2 \frac{\sqrt{(a+d)^{2}+z^{2}}}{a+d}-2 \frac{z}{a+d}-\frac{a+d}{\sqrt{(a+b)^{2}+z^{2}}}\right] .
\end{gathered}
$$

Для $\mathrm{z}=0$, линия начального контакта инструмента с дробимой породой, касательное напряжение определяется $\tau_{\mathrm{xz}}=\mathrm{q}_{\mathrm{max}}$. При нахождении напряжений вблизи поверхности соприкосновения инструмента рассматриваются зависимости при $\mathrm{z}=0: \mathrm{Sh} \alpha=0, \sin \beta 0=0$. Из выражения (2) для $\mathrm{Ch} \alpha=1$ и $\mathrm{Sh} \alpha=0$ имеем $\mathrm{x}$

$$
\begin{aligned}
& \sigma_{x}=2 q_{\max } \cos \left(\beta_{0}\right)=2 q_{\max } \frac{x}{a+d} ; \sigma_{y}=2 \mu q_{\max } \cos \left(\beta_{0}\right)=2 \mu q_{\max } \frac{x}{a+d} ; \sigma_{z}=0 ; \\
& \tau_{x z}=q_{\max } \cdot \sin \left(\beta_{0}\right)=q_{\max } \cdot \sqrt{1-\left(\frac{x}{a+d}\right)} .
\end{aligned}
$$

Если, принять $\sin \beta_{0}$ и $\cos \beta_{0}$ то из формулы (2) получим $\mathrm{x}>\mathrm{a}+\mathrm{d} . \mathrm{B}$ этом случае рассматриваются участки породы, находящиеся вне зоны контакта. Подставляя принятые значения в уравнения (1) компоненты напряжённого состояния породы и дробящих элементов будут:

$$
\begin{array}{r}
\sigma_{x}=2 q_{\max } \frac{1}{a+d}\left[\begin{array}{lll}
x & \sqrt{x^{2}(a+b)^{2}}
\end{array}\right] ; \sigma_{z}=\tau_{x z}=0 ; \\
\sigma_{y}=2 \mu q_{\max } e^{\alpha}=2 \mu q_{\max } \frac{1}{a+d}\left[\begin{array}{ll}
x & \sqrt{x^{2}(a+d)^{2}}
\end{array}\right]
\end{array}
$$

Из выражений (4 и 5) видно, что максимальные значения компоненты напряжённого состояния принимают при $\mathrm{x}= \pm(\alpha+\mathrm{d})$ (на границе зоны контакта) тогда при $\mathrm{z}=\mathrm{x}=0$ :

$$
\sigma=2 q_{\max } ; \sigma_{y}=2 \mu q_{\max } ; \tau_{x z}=q_{\max }
$$

Для оценки прочности и сопоставительного анализа принята ве-

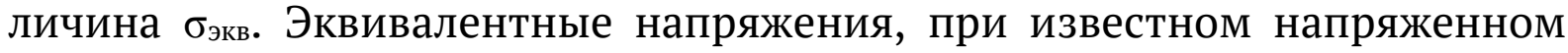
состоянии находятся с использованием главных напряжений, поэтому необходимо перейти от нормальных напряжений к главным. Зная компоненты напряжений $\sigma_{x}, \sigma_{y}, \sigma_{z}, \tau_{x y}$ с использованием известных зависимостей можно получить условие напряженности и по главным напряжениям: 
«Системні технологіï» 5 (124) 2019 «System technologies»

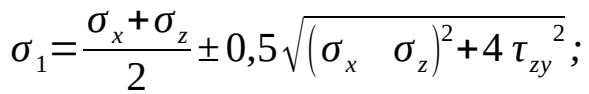

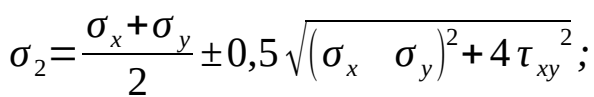

$$
\begin{aligned}
& \sigma_{3}=\frac{\sigma_{z}+\sigma_{z}}{2} \pm 0,5 \sqrt{\left(\begin{array}{ll}
\sigma_{z} & \sigma_{z}
\end{array}\right)^{2}+4 \tau_{z y}{ }^{2}} \text {. }
\end{aligned}
$$

Для рассматриваемой задачи (плоское напряжённое состояние) уравнения (7) принимают вид:

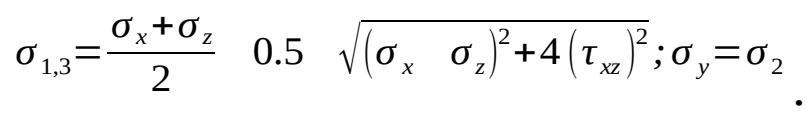

Тогда величина главных $\sigma$ по плоскости $\mathrm{x}=0$ с использованием уравнений (3) будут:

$$
\sigma_{1.3}= \pm \tau_{x z} ; \sigma_{2}=0 ;
$$

по плоскости $\mathrm{z}=0$ при $\mathrm{x}>\mathrm{a}+\mathrm{d}(5)$ :

$$
\sigma_{1}=\sigma_{x} ; \sigma_{2}=\sigma_{y} ; \sigma_{3}=0 ;
$$

по плоскости $\mathrm{z}=0$ при $\mathrm{x}<\mathrm{a}+\mathrm{d}(4)$ :

$$
\sigma_{1}=2 q_{\max }\left(\cos \beta_{0}\right), \quad \sigma_{2}=\sigma_{y}, \quad \sigma_{3}=q_{\max }\left(\cos \beta_{0}+1\right)
$$

Полученные выражения (3-5 и 8-10) описывают напряжённое состояние породоразрушающих элементов дробящего инструмента и породы в зоне их совместного контакта в случае приложения к инструменту только касательных нагрузок, равномерно распределенных по зоне контакта. На рис.3 представлены эпюры распределения напряжений по зоне контакта, полученные при $\mu=0,2$.

В вертикальной плоскости, проходящей через середину контакта, действуют $\sigma_{1}, \sigma_{2}, \tau_{\text {ху }}$ (рис.3). Характер распределения названных напряжений в вертикальной плоскости подобен. Максимальные их значения, равные qмах, имеют место на поверхности контакта $(\mathrm{z}=0)$. С глубиной (вглубь породы) эти значения интенсивно уменьшаются. Уже на расстоянии $\mathrm{z}=0,5(\mathrm{a}+\mathrm{d})$ от поверхности взаимодействия инструмента с породой действующие напряжения снижается примерно в 3 раза и составляет 0,3qmax. На глубине $\mathrm{z}=\mathrm{a}+\mathrm{d}$ они уменьшаются в 10 раз, а на расстоянии $\mathrm{z}=2(\mathrm{a}+\mathrm{d})$ от зоны контакта значения $\sigma$ и $\tau$ стремятся к нулю. 
«Системні технологіï» 5 (124) 2019 «System technologies»
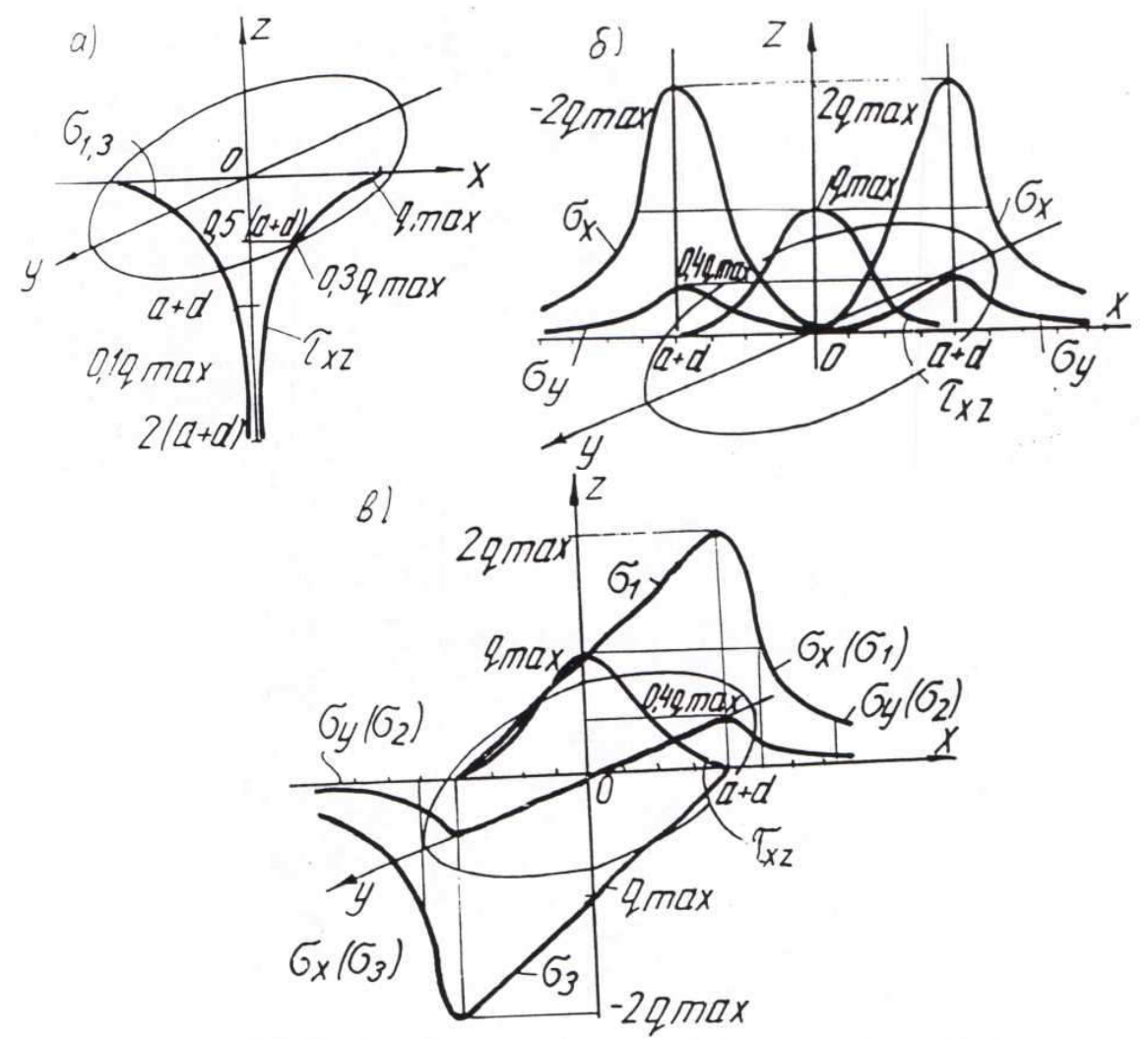

Рисунок 3 - Распределение напряжений по зоне контакта:

а) вертикальная плоскость - главные и касательные напряжения;

б) горизонтальная плоскость - нормальные и касательные напряжения;

в) горизонтальная плоскость - главные и касательные напряжения

В горизонтальной плоскости, включающей зону контакта, действуют главные, нормальные и касательные напряжения (рис.3).

Нормальное напряжение $\sigma_{x}$ в пределах площадки контакта изменяется от $\sigma_{x}=2 q_{\max }$ на границе площадки до 0 в центре ее. При проходе от центра к периферии контакта $\sigma_{x}$ интенсивно возрастают и меняют знак на обратный. На противоположной границе контакта они снова возрастают до $\sigma_{x}=-2 q_{\max }$. За пределом зоны контакта (слева и справа от нее) $\sigma_{x}$ вначале резко снижается, но затем эпюра выхолащивается и при $x>1,5(a+d)$ стремится к оси $x$, т.е. $\sigma_{x}$ стремится к нулю.

Распределение напряжений по плоскости контакта (горизонтальная плоскость) подобно рассмотренному выше с той лишь разницей, что абсолютное значение $\sigma_{y}$ меньше $\sigma_{x}$ на величину $\mu$. Закон изменения напряжений $\sigma_{x}$ и $\sigma_{y}$ одинаков, как в пределах плоскости контакта, так и вне ee. 
«Системні технології» 5 (124) 2019 «System technologies»

Касательные напряжения имеют максимальные значения $\tau_{\max }=q_{\max }$ в центре площади контакта. К границе зоны контакта они уменьшаются и принимают нулевые значения.

Главным напряжениям $\sigma_{1}, \sigma_{2}, \sigma_{3}$, действующим в горизонтальной плоскости, свойственен довольно сложный характер распределения.

Напряжение $\sigma_{1}$ по площадке контакта изменяется от $\sigma_{1}=2 q_{\text {max }}$ до 0. Нулевые значения $\sigma_{1}$ имеют со стороны действия силы $Q_{1}$. С противоположной стороны на границе контакта $\sigma_{1}$, достигают максимальных значений, которые вне площадки, интенсивно снижаются и при $x>1,5(a+d)$ приближаются к нулевым значениям. За границей контакта изменение напряжений $\sigma_{1}$ происходит подобно нормальному напряжению $\sigma_{x}$.

В рассматриваемом случае $\sigma_{2}$ распределяются по площадке контакта в полном соответствии с напряжениями $\sigma_{y}$ как по значениям действующих напряжений, так и по закону их распределения.

Для $\sigma_{3}$ в горизонтальной плоскости контакта инструмента с дробимой породой свойственен характер изменения напряжений $\sigma_{1}$, но имеющий перевёрнутое (зеркальное) отображение. Со стороны приложения нагрузки $Q_{1}$ на границе контакта $\sigma_{3}$ имеет максимальное значение $\sigma_{3}=-2 q_{\max }$ затем они по площадке контакта интенсивно снижаются и на противоположном контуре контакта достигают нулевых значений. Вне площади контакта $\sigma_{3}$ от максимальных своих значений изменяются подобно нормальным напряжениям $\sigma_{x}$. На расстоянии $x=-0,25(a+d)$ значение $\sigma_{3}$ уменьшается в 2 раза и становится равной $q_{\text {max }}$ $\left(\sigma_{x}=-q_{\max }\right)$. При дальнейшем удалении от границы контакта градиент изменения напряжения уменьшается и при $x>1,5(a+d) \sigma_{3}$ приближаются к нулю.

Необходимо иметь ввиду, что рассмотренное очень сложное распределение действующих напряжений относится к ограниченной площадке контакта, возникающей в результате взаимодействия породоразрушающих элементов дробящих плит с крепкой горной породой. За время контакта инструмента с дробимой породой в зоне их соприкосновения возникает сложное напряжённое состояние с большой концентрацией напряжений. Напряжения действуют по небольшой поверхности, состав- 
«Системні технології» 5 (124) 2019 «System technologies»

ляющей единицы мм, и изменяются с большим градиентом при проходе вдоль сечения плоскости контакта и вблизи его зоны.

Зная напряжённое состояние дробящих элементов или породы в зоне их контакта от действия нормальной нагрузки $Q$ и от касательных сил $Q_{1}$, нетрудно получить общее напряжённое состояние методом простого наложения. Оценка напряженно-деформированного состояния проводится по суммарному значению $\sigma_{э к в}$.

Наличие дополнительных внешних сил в зоне контакта сказывается на размерах зоны контакта, расстоянии участка с максимальными касательными напряжениями от поверхности контакта и на растягивающих и касательных напряжениях по зоне контакта. Действие тангенциальных сил $Q_{1}$, которые увеличиваются еще и за счет контактного трения, приводит к увеличению касательных напряжений, т.е. в рассматриваемом случае создаётся более сложное напряжённое состояние, по сравнению со схемами разрушения породы при действии только силы $Q$, что способствует эффективному разрушению пород. Этим объясняется увеличение производительности и эффективности дробления в дробилках со сложным движением щёк.

Максимальные значения контактных напряжений, по абсолютному значению, увеличиваются с ростом как нормальных, так и касательных нагрузок. При наличии $Q_{1}$ существенно возрастают значения нормальных и касательных напряжений, зарождается зона всестороннего растяжения, размеры которой и напряжения резко возрастают с увеличением касательной нагрузки. Это еще раз подтверждает вывод [9] о том, что в механизме разрушения именно эти напряжения определяют зону зарождения трещин, по линиям скольжения, которых происходит разрыв и сдвиг породы, приводящие к ее разрушению.

Рассмотрим модель формирования режимов нагружения в конусных дробилках.

Конусную дробилку можно рассматривать как результат развития щековой дробилки, связанный с переходом от плоского к пространственному движению дробящего органа (рис. 4). Дробление материала 
«Системні технології» 5 (124) 2019 «System technologies»

происходит в кольцевом рабочем пространстве между наружным неподвижным и внутренним подвижным дробящим конусом (рис. 4,a, б).

Внутренний дробящий конус может опираться на сферический подшипник скольжения, как показано на рисунке 4,б (т.н. дробилка Саймонса для среднего и мелкого дробления), или подвешиваться в верхней части неподвижного наружного конуса - т.н. гирационная дробилка для крупного дробления.

Внутренний дробящий конус приводится в движение вращением эксцентрикового стакана вокруг центральной оси наружного неподвижного конуса (т.н. оси дробилки).

При вращении эксцентрикового стакана ось дробящего конуса описывает коническую поверхность вокруг оси дробилки.

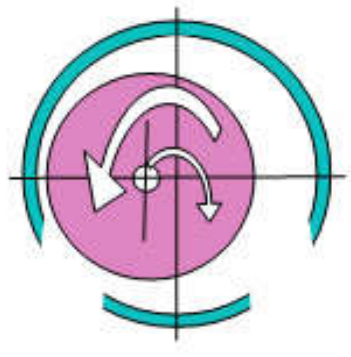

a

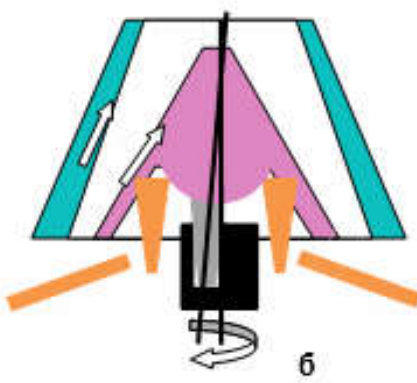

6

а) горизонтальное вращение конуса; 6) вертикальное качание конуса. Рисунок 4 - Схема конусной дробилки

В вертикальной плоскости подвижный конус совершает качательные движения (рис.4,б), как в щековой дробилке с простым движением щеки.

В горизонтальной плоскости (рис.4,a) дробящий конус совершает круговые движения внутри неподвижного конуса, последовательно приближаясь к каждой его точке. При этом сам дробящий конус катится по дробимой руде, вращаясь в направлении, противоположном вращению эксцентрикового стакана. По данным [5], в хорошо отрегулированной дробилке, подвижный конус вращается вокруг своей оси с частотой 1 рад /с в направлении вращения, а под нагрузкой - с частотой 0,315 - 0,42 рад/с - в противоположном направлении.

В конусной дробилке силы трения в вертикальной плоскости возникают на контакте породы и с внешним и с внутренним конусом. Они 
«Системні технології» 5 (124) 2019 «System technologies»

ориентированы так же, как и в щековой дробилке с простым движением щеки.

В горизонтальной плоскости суммарная проекция сил трения на контакте с дробимой рудой по дуге окружности дробящего конуса равна 0. Свободно вращающийся вокруг своей оси дробящий конус не может воспринять момента от сил трения по дуге контакта с рудой. По этой причине силы трения на контакте с дробящим конусом распределяются так же, как под плитой плоского пресса - в разные стороны (рис.5,a). А вот суммарная проекция сил трения на контакте с дробимой рудой по дуге окружности неподвижного конуса может быть и не равна 0. Потому, что внешний корпус воспринимает моменты сил трения по дуге его контакта с рудой (рис. 5,б).

Суммарная проекция нормальных усилий по контакту с поверхностью дробящего конуса должна проходить через его ось (рис.5,a), а по контакту с неподвижным конусом - через ось дробилки (рис. 5,б). Именно не коллинеарное направление нормальных усилий от неподвижного и подвижного конусов позволяет определить направление действия силы трения на контакте руды с корпусом дробилки.

На рисунке 6 показаны силы, действующие на руду со стороны конусов. Он получен совмещением рисунков 5, а и 5,б и изменением направления действия усилий на противоположное.

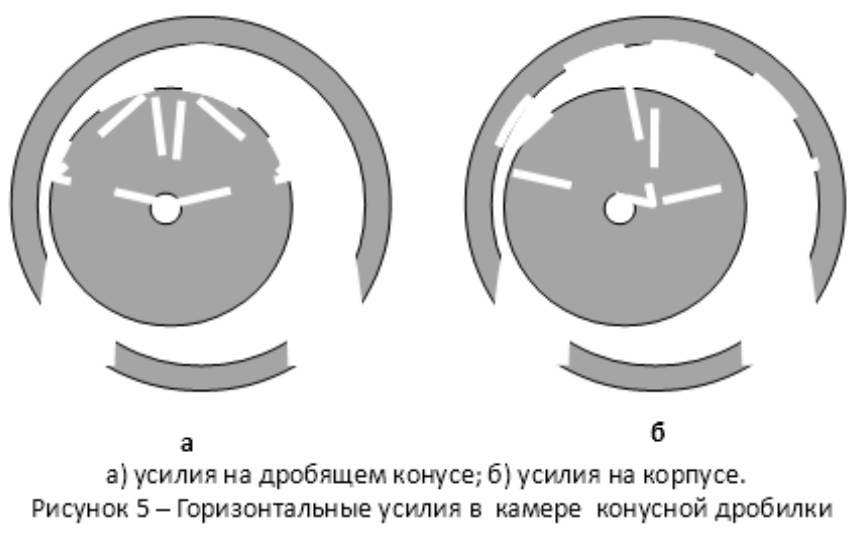

Дробление в локальных зонах левой и правой части происходит не одновременно, а последовательно. Эти зоны совмещены в одном рисунке для иллюстрации постоянства направления действия сил трения на контакте с корпусом дробилки. 
«Системні технології» 5 (124) 2019 «System technologies»

Одним из условий равновесия руды является соосность усилий от дробящего конуса и от корпуса. Они должны располагаться на одной линии. В этом случае дробимая масса уравновешена от крутящих моментов. Нетрудно определить и положение этой линии. Она совпадает с результирующей нормальной компоненты дробящего конуса (вспомним, что суммарное трение на его поверхности равно 0). Отсюда сразу следует, что суммарное усилие со стороны неподвижного конуса обязательно должно состоять не только из нормальной, но и из касательной компоненты.

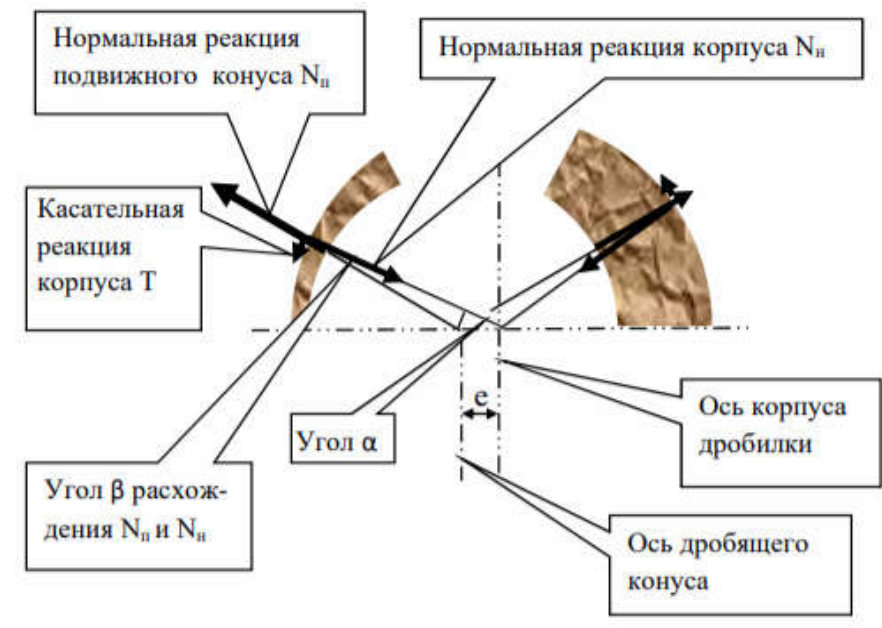

Рисунок 6 - Направление касательной компоненты на контакте руды с внешним неподвижным конусом дробилки.

Ведь нормальная компонента направлена к оси корпуса и не может совпадать с направлением усилия от дробящего конуса, направленного к оси последнего.

«Укладывает» реакцию корпуса на линию реакции дробящего конуса касательная компонента $T$, направленная против направления вращения эксцентрика как в узкой, так и в широкой части рабочей камеры. Ее величина определяется из двух очевидных тригонометрических соотношений:

$$
\begin{gathered}
T / N_{H}=\operatorname{tg} \beta \\
\operatorname{tg} \beta=e \sin \alpha /(R-e \cos \alpha),
\end{gathered}
$$

в которых $R$ - радиус внешнего неподвижного конуса, е - эксцентриситет оси подвижного конуса.

Исключив из этих выражений $\operatorname{tg} \beta$, получим:

$$
T=N_{H} e \sin \alpha /(R-e \cos \alpha)
$$


«Системні технологіï» 5 (124) 2019 «System technologies»

Эксцентриситет оси подвижного конуса дробилок составляет $(1,5 \div 3) * 10^{-2} \mathrm{M}$, а радиус неподвижного конуса - $(60 \div 120) * 10^{-2}$ м. С учетом этих величин соотношение (13) дает оценку максимальной касательной компоненты $T$ на уровне $0,025 N_{н}$. Это почти на порядок меньше силы трения скольжения руды об футеровку дробилки, которая является единственной причиной возникновения касательной компоненты.

Парадокс можно объяснить только тем, что и на контакте с неподвижным корпусом силы трения имеют встречное направление. Т.е. силы трения слева и справа от результирующей нормальной компоненты направлены в разные стороны.

Осталось выяснить вопрос об ориентации сил трения на корпусе по отношению к направлению вращения эксцентрика. В строгой постановке для этого необходимо решить контактную задачу для сыпучей среды между криволинейными эксцентрично расположенными поверхностями.

Чтобы избежать сложных и громоздких вычислений для определения ориентации сил трения мы рассмотрим модель рудной массы в виде одного крупного куска руды в форме сплющенного треугольника.

Кусок касается конусов только в 3 точках. Средняя часть основания опирается на подвижный, а края - на неподвижный конусы, как показано на рисунке 7,а.

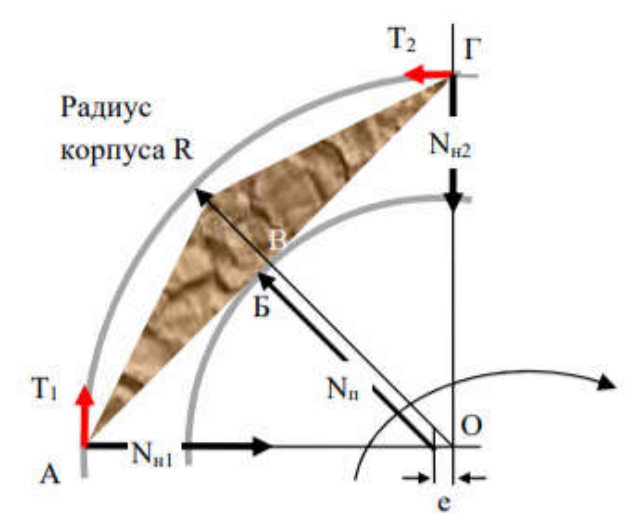

a

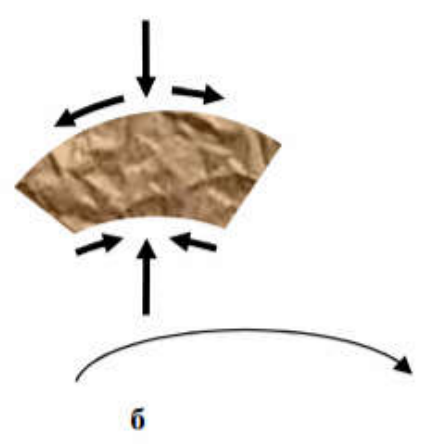

6

а) не верное направление сил трения; б) верное направление сил трения.

Рисунок 7 - Направление сил трения на контакте с дробимой рудой

Направление силы трения $T_{1}$ слева от результирующей нормали принято совпадающим с направлением вращения эксцентрика. 
«Системні технологіï» 5 (124) 2019 «System technologies»

Сила $T_{2}$ справа от результирующей нормали будет направлена в противоположную сторону - против направления вращения эксцентрика.

Поскольку кусок руды находится в равновесии, сумма моментов сил тносительно точки А должна быть равна нулю:

$$
N_{H 2} A O-T_{2} \Gamma O-N_{n} A B=0 .
$$

Такое же условие можно записать и для точки $\Gamma$ :

$$
N_{H 1} \Gamma O-T_{1} A O-N_{n} Б \Gamma=0 .
$$

Учитывая, что $A O=\Gamma O=R ; T_{1}=f N_{H 1} u T_{2}=f N_{H 2}$,

где $f$ - коэффициент трения, а также, что $A B=B \Gamma=A \Gamma / 2=R / \sqrt{2}$,

определим плечи АБ и БГ

$$
\begin{aligned}
& A Б=(R-e) / \sqrt{2} ; \\
& Б \Gamma=(R+e) / \sqrt{2},
\end{aligned}
$$

и подставляя эти соотношения в $(14,15)$, получим:

$$
\begin{gathered}
N_{H 2} R-f N_{H 2} R-N_{n}(R-e) / \sqrt{2}=0, \\
N_{H 1} R-f N_{H 1} R-N_{n}(R+e) / \sqrt{2}=0, \\
\text { или } N_{H 2}=N_{n}(1-e / R) /[\sqrt{2}(1-f)], \\
N_{H 1}=N_{n}(1+e / R) /[\sqrt{2}(1-f)] .
\end{gathered}
$$

Сравнивая формулы (22) и (23), мы видим, что нормальная реакция на левый угол рудного треугольника $N_{н 1}$ больше нормальной реакции на его правый угол $N_{н 2}$ за счет разного знака слагаемого $e / R$.

Тогда, согласно (16), и сила трения на левом краю больше, чем на правом:

$$
T_{1}>T_{2}
$$

а их равнодействующая $T$ направлена в сторону вращения эксцентрика. Но из выражения (13) и рисунка 6 следует, что направление $T$ противоположно направлению вращения эксцентрика.

Значит, направления действия сил трения $T_{1}$ и $T_{2}$ на рис. 7 , а было выбрано не верно (поэтому они показаны красным цветом) и его нужно сменить на противоположное, как показано на рис. 7,б. В уравнениях (14 и 15) нужно изменить знаки при $T_{1}$ и $T_{2}$. Тогда уравнения (22 и 23) примут вид: 
«Системні технологіï» 5 (124) 2019 «System technologies»

$$
\begin{gathered}
N_{H 2}=N_{n}(1-e / R) /[\sqrt{ } 2(1+f)] \\
N_{H 1}=N_{n}(1+e / R) /[\sqrt{2}(1+f)],
\end{gathered}
$$

а результирующая касательная компонента $T$ будет направлена противоположно направлению вращения эксцентрика.

При этом и левее, и правее линии нормальной реакции направление силы трения руды об корпус противоположно направлению ее силы трения об дробящий конус.

Этот вывод, полученный для модели в виде одного крупного куска в форме сплющенного треугольника, мы распространяем на реальную рудную массу, заполняющую частично или полностью камеру дробилки (рис.7,б).

Таким образом, вращение дробящего конуса обеспечивает дробление руды в эффективном режиме скользящего сжатия.

Обсуждение результатов. Режим скользящего сжатия в конусной дробилке сопровождается намного меньшими потерями энергии, чем в щековой. Из-за взаимного погашения усилий трения, их результирующая, определяемая по формуле (13), снижается на порядок.

Создание более эффективного режима использования фрикционных характеристик обеспечило более низкую энергоемкость дробления [10]. Лучшая энергоемкость, наряду с другими преимуществами технологического и эксплуатационного характера, основное из которых - возможность работы под завалом привела к почти полному вытеснению щековых дробилок конусными в железорудной промышленности.

Конусные дробилки в железорудной промышленности преобладают по распространенности не только над щековыми, но и над валковыми, если речь идет о крупности дробления до $(10 \div 15) * 10^{-3} \mathrm{M}$.

\section{Выводы}

1. Анализ эпюр распределения напряжений по зоне контакта позволяет сделать следующее заключение. С увеличением касательной нагрузки уменьшается зона всестороннего сжатия материала в зоне контакта, глубина распределения наиболее напряжённой точки ( $\left.\tau_{\max }\right)$ уменьшается и с увеличением Q1 приближается к поверхности контакта. Имеет место значительная зона действия растягивающих и сдвигающих напря- 
«Системні технологіï» 5 (124) 2019 «System technologies»

жений, которые и являются решающими фактором при разрушении в условиях так называемого скользящего сжатия.

2. При сложном движении щеки она перемещается не только в радиальном, но и в тангенциальном направлении - вдоль действия силы трения. Именно вредная работа силы трения на тангенциальном перемещении «съедает» значительную часть эффекта от скользящего сжатия и приводит к повышенному износу футеровки по сравнению с простым движением щеки в радиальном направлении. Тем не менее, доказанное практикой эксплуатации преимущество в производительности дробилок со сложным движением щеки подтверждает полезность и правильность разработанных математических моделей влияния фрикционных характеристик на процессы разрушения.

3. Напряженное состояние породы в конусных дробилках также соответствует модели более эффективного скользящего сжатия. При этом, в конусных дробилках отсутствует вредная работа сил трения, а их действие только повышает эффективность разрушения.

4. Приведённая математическая модель механизма распределения напряжений с учетом внешнего трения позволяет управлять эффективностью процесса разрушения при соответствующих инженерных расчётах щековых и конусных дробилок.

\section{ЛИТЕРАТУРА / ЛІТЕРАТУРА}

1. Блохин В.С., Большаков В.И., Малич Н.Г. Основные параметры технологических машин. Машины для дезинтеграции твёрдых материалов: Учебное пособие. - Днепропетровск: ИМА-пресс, 2006.-404c.

2. Малич Н.Г. Научные основы расчёта параметров машин для земляных работ в горно-металлургическом комплексе: Монография. Днепропетровск: ИМА-пресс, 2010. -308с.

3. Н.Г. Малич, Л.М. Васильев, О.А. Усов Исследование влияния параметров нагружения на распределение напряжений, действующих при разрушении шихтовых материалов в дробилках // Металлург. и горнорудн. пром-сть.-2015.-№7.-С.99-106.

4. Перов В.А., Андреев Е.Е., Биленко Л.Ф. Дробление, измельчение и грохочение полезных ископаемых.-М.: Недра, 1990.-310 с.

5. Клушанский Б.В., Косарев А.И., Муйземнек Ю.А. Дробилки. Конструкция, расчёт, особенности эксплуатации. - М.: 'Машиностроение”, 1990. 320c.

6. Штаерман И.Я. Контактная задача теории упругости. - M. - JL: Гостехиздат, 1949. - 276с. 
«Системні технологіï» 5 (124) 2019 «System technologies»

7. Лейбензон Л.С. Курс теории упругости. - М. - Л.: Гостехиздат, 1977.

8. Расчёты на прочность в машиностроении./ Под ред. С.Д. Пономарева. М.: Машгиз, 1959.

9. Васильев Л.М., Васильев Д.Л., Малич Н.Г., Ангеловский А.А. Механика образования форм разрушения образцов горных пород: Монография.Днипро, ИМА-пресс.-2018-172с.

10. Бедрань Н.Г. Машины для обогащения полезных ископаемых / Н.Г. Бедрань.- К. :Высшая школа, 1980.-416с.

\section{REFERENCES}

1. Blokhin V.S., Bolshakov V.I., Malich N.G. Osnovnye parametry tekhnologicheskikh mashin. Mashiny dlya dezintegraczii tvyordykh materialov: Uchebnoe posobie. - Dnepropetrovsk: IMA-press, 2006.-404s.

2. Malich N.G. Nauchnye osnovy raschyota parametrov mashin dlya zemlyanykh rabot $\mathrm{v}$ gorno-metallurgicheskom komplekse: Monografiya. Dnepropetrovsk: IMA-press, 2010. -308s.

3. N.G. Malich, L.M. Vasilev, O.A. Usov Issledovanie vliyaniya paramet-rov nagruzheniya na raspredelenie napryazhenij, dejstvuyushhikh pri raz-rushenii shikhtovykh materialov v drobilkakh // Metallurg. i gornorudn. prom-st .2015.-\#7.-S.99-106.

4. Perov V.A., Andreev E.E., Bilenko L.F. Droblenie, izmel'chenie i grokhochenie poleznykh iskopaemy`kh.-M.: Nedra, 1990.-310 s.

5. Klushanskij B.V., Kosarev A.I., Mujzemnek Yu.A. Drobilki. Konstruk-cziya, raschyot, osobennosti ekspluataczii. - M.: "Mashinostroenie", 1990. - 320s.

6. Shtaerman I.Ya. Kontaktnaya zadacha teorii uprugosti. - M. - JL: Gostekhizdat, 1949. - 276s.

7. Lejbenzon L.S. Kurs teorii uprugosti. - M. - L.: Gostekhizdat, 1977.

8. Raschyoty na prochnost v mashinostroenii./ Pod red. S.D. Ponomareva. M.: Mashgiz, 1959.

9. Vasilev L.M., Vasilev D.L., Malich N.G., Angelovskij A.A. Mekhanika obrazovaniya form razrusheniya obrazczov gornykh porod: Monografiya.Dnipro, IMA-press.-2018-172s.

10. Bedran N.G. Mashiny dlya obogashheniya poleznykh iskopaemykh / N.G. Bedran.- K. :Vysshaya shkola, 1980.-416s.

Received 18.11.2019. Accepted 21.11.2019.

Моделювання розподілу напружень що діють при руйнуванні порід в дробарках 3 урахуванням контактного тертя

Наведено дослідження розподілу напружень, що діють при руйнуванні міцних матеріалів в зоні контакту з бронеплитами в щокових дробарках зі складним рухом щоки $і$ з конусами в конусних дробарках. Проаналізовано вплив напрямку сил контактного тертя при стисненні на ефективність руйнування гірської маси. Розроблено математичну мо- 


\section{«Системні технології» 5 (124) 2019 «System technologies»}

дель для визначення напруженного стану породи. Показана можливість керування режимами навантаження для підвищення ефективності процесу дроблення.

\section{Simulation of tensions distribution that works in destruction of rocks in crushers with contact friction}

Studies of stress distribution, acting during the destruction of solid materials in the zone of contact with the armor plates in jaw crushers with complex movement of the jaw and cones in cone crushers are made.

Analysis of plots of the stress distribution in the contact zone leads to the following conclusion. With the increase of the tangential load, the zone of the material compression decreases, in the contact zone the distribution depth of the most tense point with the shear stresses decreases and with the increase of shear stress it approaches the surface of contact. There is considerable coverage of tensile and shear stresses, which are the decisive factor in the destruction in terms of the so-called sliding compression.

The influence of the force directions of the contact friction during the compression efficiency of destruction of rock mass. When the complex movement of the jaws it is moved not only in a radial but in a tangential direction along the force of friction. That is a bad work of the friction force on the tangential displacement "eats" a considerable part of the effect of moving the compression and leads to increased wear of the lining compared with a simple movement jaws in the radial direction. However, the proven practice of exploiting the performance advantages of crushers with complex movement of the jaws confirms the usefulness and correctness of the developed mathematical models of the influence of friction characteristics on fracture.

The stress state of the rock in cone crushers also matches the model of more efficient sliding compression. Thus, there is no harmful the work of the friction forces in cone crushers, and their action only increases the efficiency of destruction.

The mathematical model of the mechanism of stress distribution with consideration of external friction allows to control the efficiency of the destruction process in appropriate engineering calculations of jaw and cone crushers.

Малич Николай Григорьевич - к.т.н., доцент, Национальная металлургическая академия Украины, Украина.

Маліч Микола Григорович - к.т.н., доцент, Національна металургійна академія України, Україна.

Malich Mykola Grigorovich - Candidate of Technical Sciences, Associate Professor, National Metallurgical Academy of Ukraine, Ukraine. 